\title{
Rapid progression of sarcomatoid carcinoma of the common bile duct
}

\author{
Moon Jin Kim', Jun Suh Lee', Yu Mi Kim', Soyeon An², Young Chul Yoon' \\ Departments of 'Surgery and ${ }^{2}$ Pathology, Incheon St. Mary's Hospital, College of Medicine, The Catholic University of Korea, Incheon, Korea
}

Since sarcomatoid carcinoma in the common bile duct (CBD) is rarely reported, the clinical course and prognosis after surgery are unclear. We report a case of a patient who died within 1 month after surgery due to rapid tumor progression. A 65-year-old woman had abdominal pain with jaundice. She was diagnosed with CBD cancer and underwent pancreatoduodenectomy. Pathologic examination revealed sarcomatoid carcinoma. There was no postoperative complication, but multiple liver metastasis was diagnosed on computed tomography at 7 days after surgery. Also, the patient complained of abdominal pain and had jaundice with elevated liver enzyme on the 14th postoperative day. Her general condition was getting worse and she died of hepatic failure 23 days after surgery. We report a case of sarcomatoid carcinoma of the $\mathrm{CBD}$ that progressed very rapidly. Further research and case reports are needed to establish proper diagnostic and treatment tools.

Keywords: Carcinosarcoma, Common bile duct, Pancreatoduodenectomy, Prognosis

\section{INTRODUCTION}

Sarcomatoid carcinoma is a rare malignant spindle cell neoplasm in which epithelial differentiation may be demonstrated by immunohistochemical or ultrastructural studies [1,2]. These tumors have been reported in many different organs, including esophagus, lung, liver, stomach, gallbladder, pancreas, small intestine, urinary tract, and uterus. Though well defined, histopathological sarcomatoid carcinoma occurring in the common bile duct (CBD) is extremely rare; and its clinical behavior and prognosis are unclear. Generally, sarcomatoid carcinoma is known to have poorer prognosis than carcinoma. There is a few report where bile duct sarco-

Received: May 18, 2019 Revised: Jun 8, 2019 Accepted: Jun 10, 2019 Correspondence to: Young Chul Yoon

Department of Surgery, Incheon St. Mary's Hospital, College of Medicine, The Catholic University of Korea, 56 Dongsu-ro, Bupyeong-gu, Incheon

21431, Korea

Tel: +82-32-280-5862, Fax: +82-32-280-5988

E-mail:k07yyc@catholic.ac.kr

ORCID: Moon Jin Kim (https://orcid.org/0000-0002-5404-3431), Jun Suh Lee (https://orcid.org/0000-0001-9487-9826), Yu Mi Kim (https://orcid.org/0000-00022811-2106), Soyeon An (https://orcid.org/0000-0003-1193-8216), Young Chul Yoon (https://orcid.org/0000-0002-5341-2370)

Copyright @ 2019 Korean Society of Surgical Oncology

This is an Open Access article distributed under the terms of the Creative Commons Attribution Non-Commercial License (http://creativecommons.org/licenses/by-nc/4.0) which permits unrestricted non-commercial use, distribution, and reproduction in any medium, provided the original work is properly cited. matoid carcinoma has better prognosis than in gallbladder carcinoma [3]. We report a case of sarcomatoid carcinoma in the CBD that progressed rapidly after surgery.

This case report was approved by the Institutional Review Boards of Catholic University Incheon St. Mary's Hospital (IRB No. OC19ZESI0026) and written in accordance with the principles of the Declaration of Helsinki. Written informed consent was obtained.

\section{CASE REPORT}

A 65-year-old female patient was visited for further management of jaundice with vague abdominal pain. She had elevated liver enzymes (total bilirubin, $7.9 \mathrm{mg} / \mathrm{dL}$; aspartate aminotransferase, 231 $\mathrm{U} / \mathrm{L}$; alanine aminotransferase, $311 \mathrm{U} / \mathrm{L}$; alkaline phosphate, 143 $\mathrm{U} / \mathrm{L})$. Her tumor marker levels were mildly elevated in carbohydrate antigen 19-9 (50.5 U/mL) and otherwise normal (alpha-fetoprotein, $2.85 \mathrm{ng} / \mathrm{mL}$; carcinoembryonic antigen, $1.16 \mathrm{ng} / \mathrm{mL}$ ). Contrast enhanced chest-abdomen-pelvis computed tomography (CT) and endoscopic retrograde cholangiopancreatography (ERCP) were performed for diagnosis and oncologic staging. CT and ERCP showed longitudinal narrowing in mid CBD (Fig. 1). Brush cytology smears from the distal bile duct showed atypical cell, suggesting adenocarcinoma. We did not perform liver magnetic resonance imaging because there was no suspicion of liver metastasis on CT. Preoperative imaging stage was T2 or T3N0M0. 

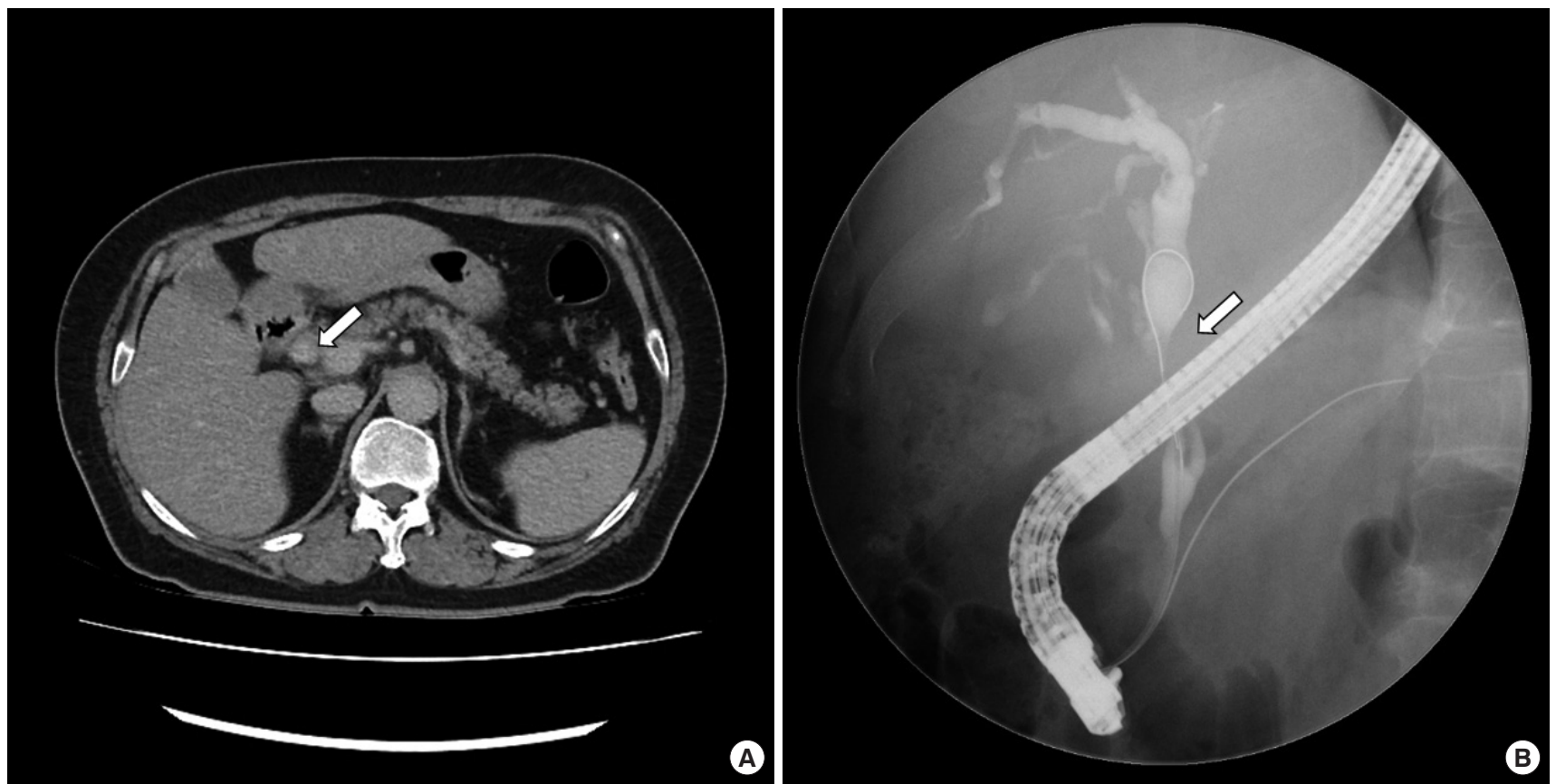

Fig. 1. (A, B) Computed tomography and endoscopic retrograde cholangiopancreatography (before surgery). White arrows illustrate bile duct sarcomatoid carcinoma, which protrudes longitudinally on bile duct.

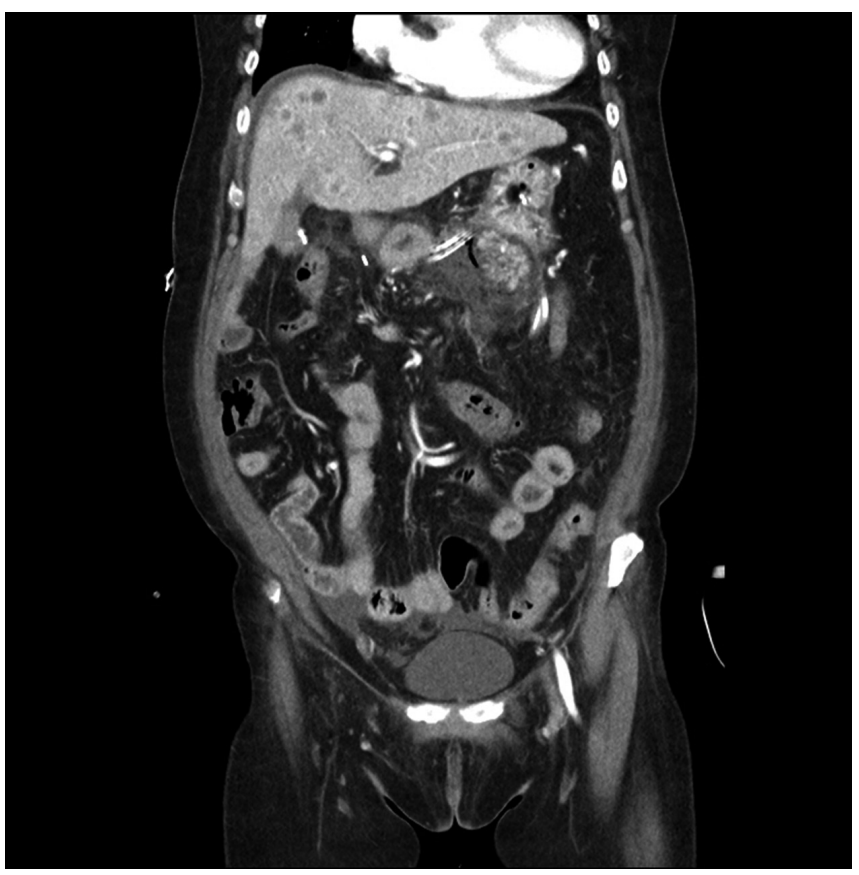

Fig. 2. Computed tomography (1 week after surgery). Multiple liver metastases were found in abdominal computed tomography.

Two weeks after CT scan, we performed pancreatoduodenectomy. Intraoperatively, there were no suspicious findings of liver metastasis. The tumor in the specimen showed an infiltrative growth pattern and the size of the tumor was measured as $3.5 \times 2.2 \times 1.5$ $\mathrm{cm}$. Microscopic findings and special staining confirmed sarcomatoid carcinoma. There were no cancer cells in the margins of the retrieved specimen, and no cancer cells were found among the 17 removed lymph nodes. Her postoperative course was uneventful. However, multiple liver masses were found in abdominal CT, scanned routinely 1 week after surgery (Fig. 2). Liver biopsy confirmed liver metastasis. Because it was only 1 week after surgery, chemotherapy was not considered. The patient gradually became malnourished and complained of generalized pain. After 2 weeks post-surgery, liver enzyme elevated gradually and we performed CT scans repeatedly. There were multiple lung metastases as well as more aggravated multiple liver metastases (Fig. 3). Her condition worsened and she died on the 23rd day after surgery due to hepatic failure.

Grossly, the tumor was a poorly-demarcated and periductal infiltrative mass, located in the distal CBD and extended to periductal soft tissue, pancreatic parenchyma, and peripancreatic soft tissue. Microscopically, the tumor was composed of components with variable histologic features. Adenocarcinoma component was focally identified with poorly-formed glandular structures and intracellular mucin (Fig. 4A). The majority of tumor cells were sarcomatoid; haphazardly arranged spindle cells in collagenous or myxoid background (Fig. 4B). Undifferentiated pleomorphic cells, poorly cohesive oval to epithelioid cells (Fig. 4C) and compact- 

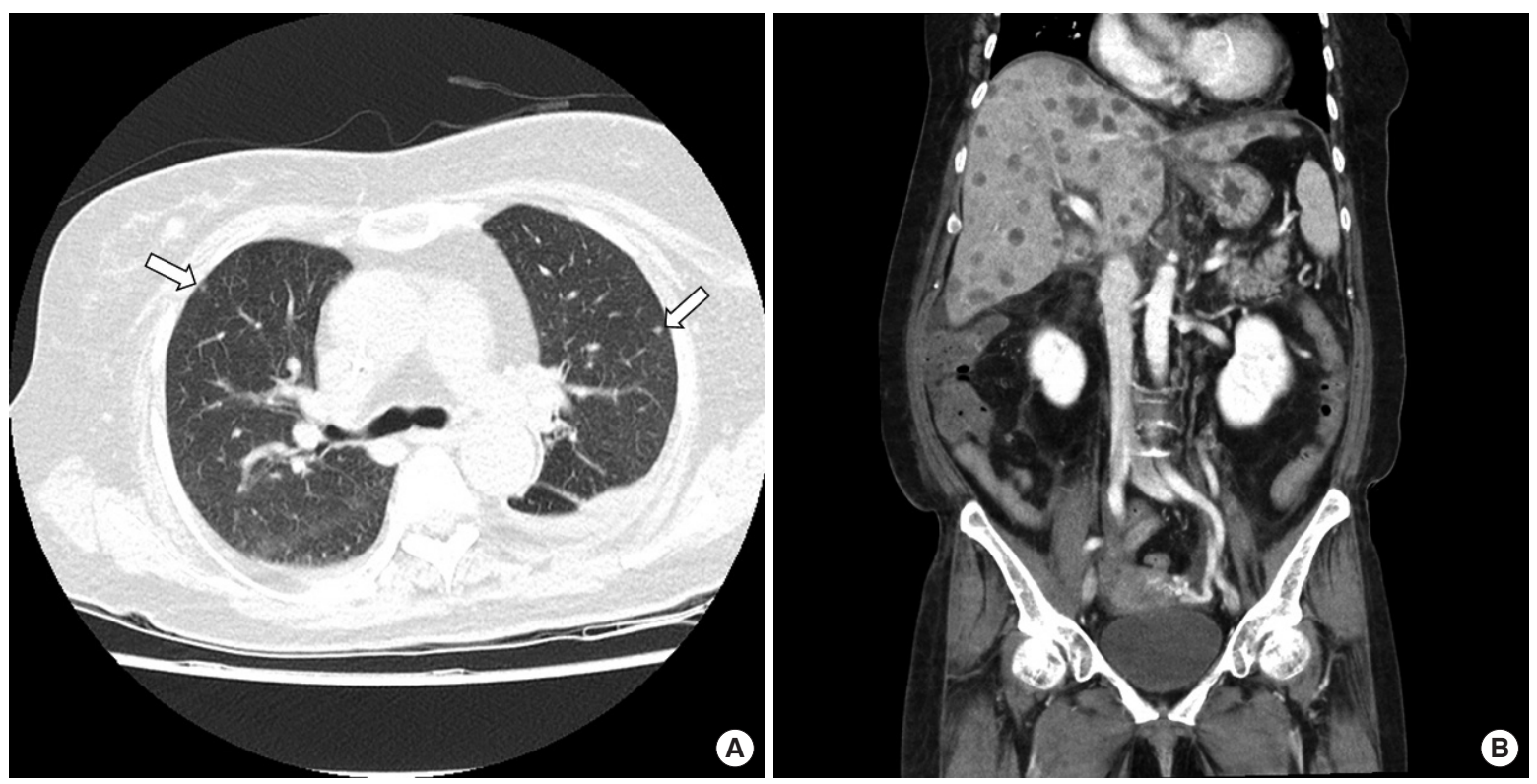

Fig. 3. Computed tomography (2 weeks after surgery). (A, B) Multiple lung and liver metastases were found in computed tomography. White arrows illustrate lung metastases.

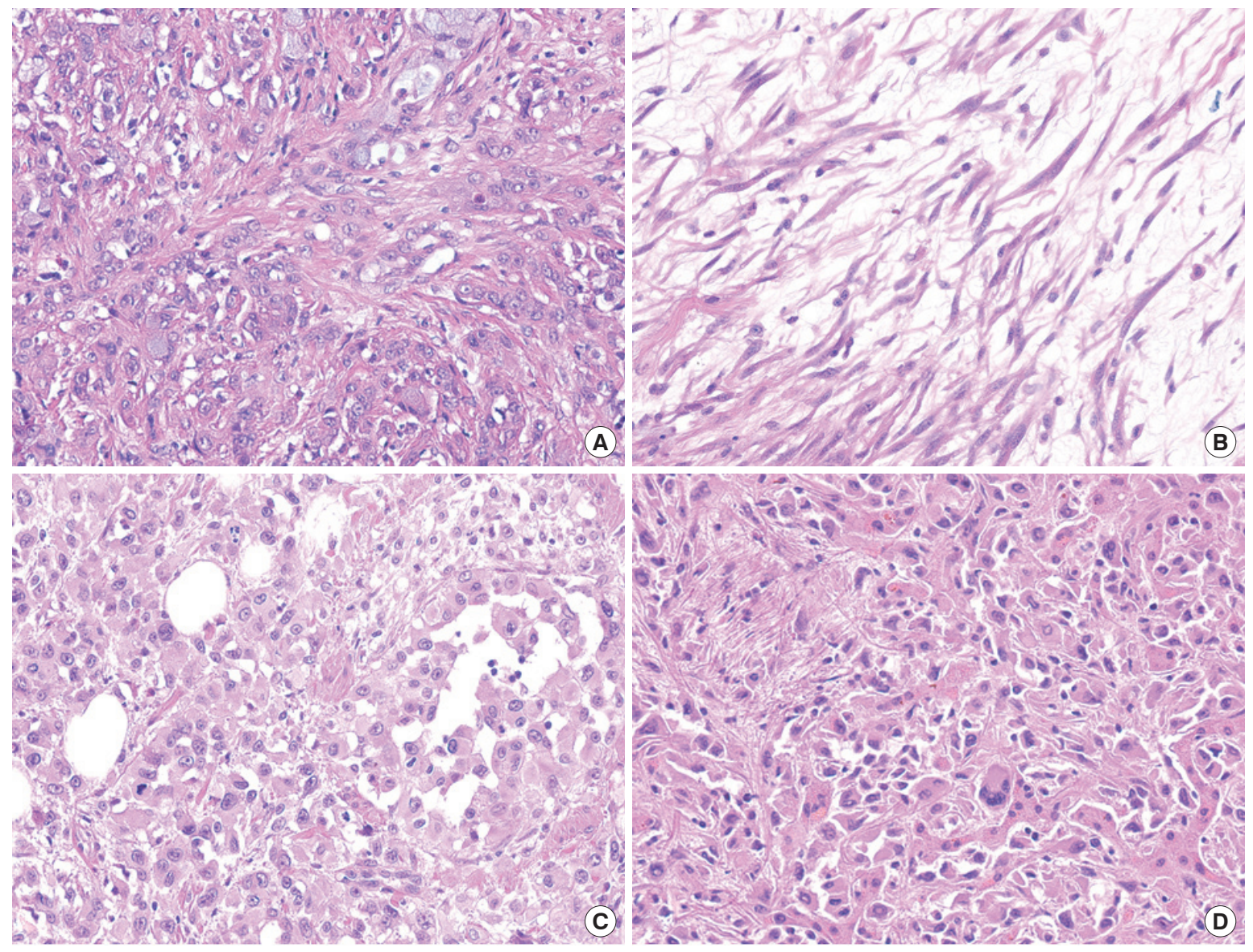

Fig. 4. Representative images of the tumor components. (A) Poorly-differentiated adenocarcinoma component with glandular structures and intracellular mucin, (B) spindle cell component with myxoid background and (C) oval and epithelioid component are observed. (D) In metastasis to liver, infiltrative tumor cells with multinucleated giant cell are observed $\left(H \& E_{1} \times 200\right)$. 

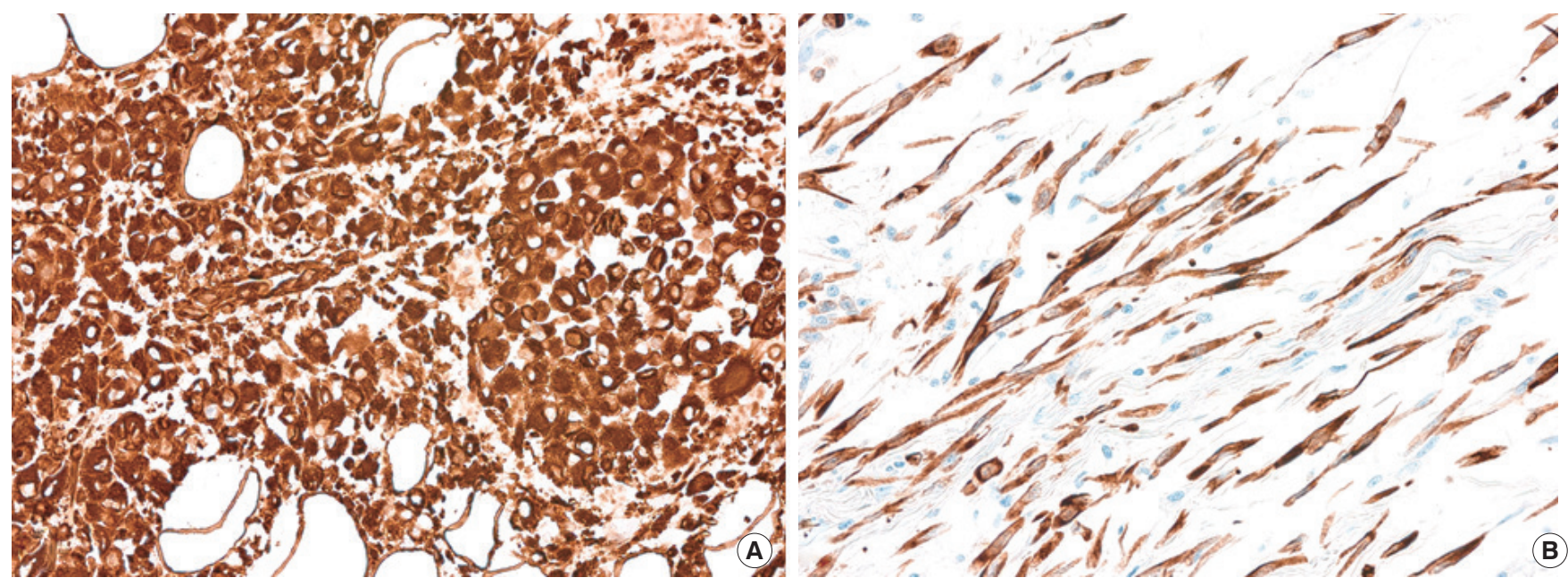

Fig. 5. Immunohistochemical staining (×200). Vimentin positivity in oval and epithelioid component (A) and pan-cytokeratin positivity in spindle cell component (B).

Table 1. Summary of clinicopathological characteristics in literature and current case

\begin{tabular}{|c|c|c|c|c|c|c|c|}
\hline Author (year) & $\begin{array}{c}\text { Sex/age } \\
(y r)\end{array}$ & Location & $\begin{array}{c}\text { Growth pattern } \\
\text { (size) }\end{array}$ & Surgery & LN status & Recurrence/site & Prognosis \\
\hline Loud et al. 1997 [6] & $\mathrm{F} / 35$ & Hilar & Polypoid (NR) & $\mathrm{BD}$ resection & NR & NR & NR \\
\hline Yoon et al. 2004 [7] & $\mathrm{M} / 78$ & Mid CBD & Infiltrative $(4.0 \mathrm{~cm})$ & PD & $2 / 22$ & NR & Died due to cardiac problem (5POD) \\
\hline Kadono et al. 2005 [8] & $\mathrm{F} / 75$ & Mid CBD & Infiltrative (NR) & PD & NR & Local recurrence & Died on 2 years due to local recurrence \\
\hline Jang et al. 2005 [3] & $\mathrm{F} / 68$ & Distal CBD & Polypoid $(3.5 \mathrm{~cm})$ & PD & NR & No recurrence & 1-Year DFS \\
\hline Sodergren et al. 2005 [9] & $\mathrm{F} / 64$ & Hilar & Polypoid $(2.0 \mathrm{~cm})$ & $\mathrm{BD}$ resection & NR & No recurrence & 5-Year DFS \\
\hline Aurello et al. 2008 [10] & $\mathrm{F} / 73$ & Distal CBD & Polypoid $(2.6 \mathrm{~cm})$ & PD & NR & No recurrence & 7-Month DFS \\
\hline Tanaka et al. 2012 [11] & $\mathrm{M} / 71$ & Distal CBD & Polypoid $(7.5 \mathrm{~cm})$ & PD & NR & Liver metastasis & Died on 10 months due to recurrence \\
\hline Kumei et al. 2015 [12] & $\mathrm{F} / 73$ & Mid CBD & Polypoid $(6.5 \mathrm{~cm})$ & PD & NR & Liver metastasis & Died on 6 months due to recurrence \\
\hline Lee et al. 2016 [13] & $\mathrm{F} / 91$ & Hilar & Polypoid $(2.0 \mathrm{~cm})$ & $\mathrm{BD}$ resection & NR & Recurrence & Died on 2 months due to recurrence \\
\hline Zhang et al. 2017 [14] & $\mathrm{F} / 51$ & Distal CBD & Infiltrative $(3.8 \mathrm{~cm})$ & PD & $0 / 12$ & No recurrence & 3-Year DFS \\
\hline Kim et al. (current study) & F/65 & Mid CBD & Infiltrative $(3.5 \mathrm{~cm})$ & PD & $0 / 17$ & Liver metastasis & Died on 23 days due to recurrence \\
\hline
\end{tabular}

$L N$, lymph node; F, female; $M$, male; NR, not reported; BD, bile duct; $C B D$, common bile duct; PD, pancreatoduodenectomy; POD, postoperative day; DFS, disease-free survival.

ly-arranged clear cells were also frequently observed. Adenocarcinoma component was focally identified with poorly-formed glandular structures and intracellular mucin (Fig. 4A). Multinucleated giant cells were observed in liver metastasis (Fig. 4D). However, no heterologous sarcomatous elements were identified.

On immunohistochemistry, tumor cells showed diffuse and strong positivity for both pan-cytokeratin and vimentin, regardless of histologic features (Fig. 5), while showing total negativity for desmin and smooth muscle actin.

\section{DISCUSSION}

Sarcomatoid carcinoma is an unusual malignant tumor and its clinical behavior has not yet been fully established. It is often con- fused with carcinosarcoma. Histologically, sarcomatous carcinoma and carcinosarcoma are completely different entities, but they are often confused because they exhibit similar histological features and clinical behavior [4]. They have aggressive clinical course characterized by local recurrences and metastasis.

Similar to carcinosarcoma in other organs, carcinosarcoma in the gallbladder also has a very poor prognosis. Median survival after surgical resection was 7 months and the reported 1-, 2-, and 3-year survival rates after surgery were $37.2 \%, 31.0 \%$, and $31.0 \%$, respectively [5]. Sarcomatoid carcinoma and carcinosarcoma of the bile duct represents very rare prevalence. To date, only 10 case reports have been reported of sarcomatoid carcinoma and carcinosarcoma in bile ducts in the English literature (Table 1) [3,6-14]. Tumors in the CBD may have a better prognosis owing to their 


\section{$\mathrm{KJCO}$}

KOREAN JOURNAL OF CliniCAL ONCOLOGY

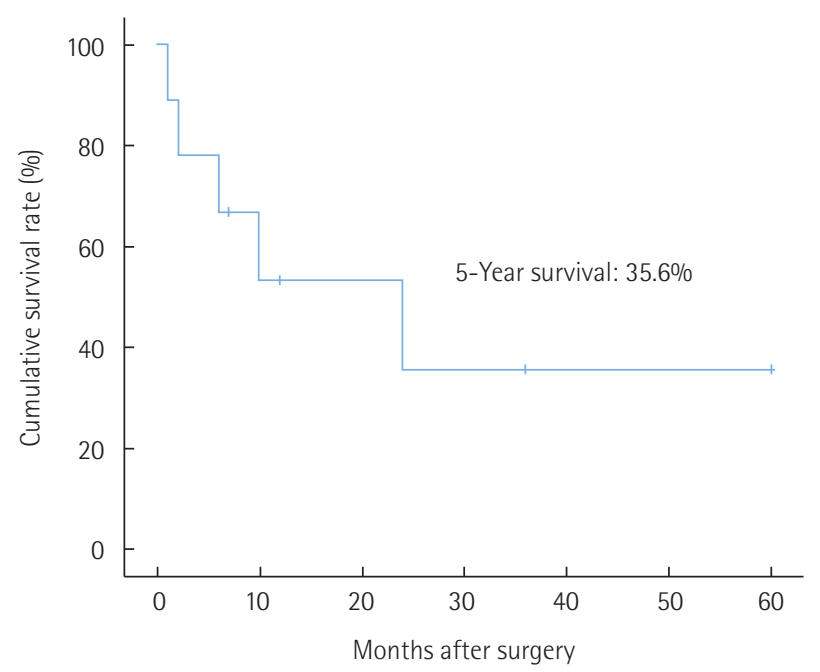

Fig. 6. Survival after surgical resection for sarcomatoid carcinoma and carcinosarcoma of the bile duct $(n=11)$.

polypoid nature that leads to earlier presentation as it obstructs the bile duct-in contrast to gallbladder sarcomatoid carcinoma, which usually presents as an infiltrative form $[3,13]$. We calculated the survival rate of bile duct sarcomatoid carcinoma and carcinosarcoma using cases reported so far. The overall 1-, 2-, and 3-year survival rates after surgery were $53.3 \%, 35.6 \%$ and $35.6 \%$, respectively (Fig. 6). It showed better survival than gallbladder cases. However, prognosis was worse than other bile duct carcinoma. In almost all cases, where there were bile duct obstructive symptoms, early detection was possible, and survival rate was improved, as mentioned. But as for recurrence, bile duct sarcomatoid carcinoma and carcinosarcoma presents very poor prognosis.

Throughout the reported case, we found recurrence within 2 months, and tumor progression was very rapid. However, there were no cases that progressed as rapidly as shown in our case. The cases reported so far mostly presented a polypoid pattern of the tumor in the CT or the pathology of the removed specimen (7 of 10 cases). Alternatively, in the present case, the tumor was flat on the bile duct and tumor growth showed an infiltrative pattern, usually shown in gallbladder carcinosarcoma. An infiltrative pattern may have poor prognosis compared with a polypoid pattern because polypoid types tend to arise earlier with obstruction of the bile duct and obstructive symptoms, which enable easier detection [14]. The present case shows very radical progression, thus having the possibility that multiple small hidden malignancies would go undetected preoperatively, as well as the histochemical features of a tumor, especially sarcomatoid cells, associated with poor prognosis. There are few references which support the reliability of chemotherapy and radiotherapy in cases of biliary sarcomatoid carcinoma [15]. At present, the first treatment option for sarcomatoid carcinoma and carcinosarcoma is surgery and complete resection of the tumor, similar to biliary carcinoma. However, as shown in this case, very poor prognosis and rapid progression to systemic metastasis proved that surgical resection is not adequate for radical cure in cases of biliary sarcomatoid carcinoma. Therefore, another treatment option should be researched, studied, and established. Still, however, the amount of cases of bile duct sarcomatoid carcinoma is tiny. Thus, more research and literature may be needed to establish proper diagnostic methods and treatment tools.

Sarcomatoid carcinoma of the bile duct may progress more rapidly than other carcinomas. However, there is currently no treatment other than complete surgical resection for as a cure. In preoperative examination, infiltrating type bile duct sarcomatoid carcinoma should be considered for rapid tumor progression after surgery.

\section{CONFLICT OF INTEREST}

No potential conflict of interest relevant to this article was reported.

\section{REFERENCES}

1. Wick MR, Brown BA, Young RH, Mills SE. Spindle-cell proliferations of the urinary tract: an immunohistochemical study. Am J Surg Pathol 1988;12:379-89.

2. Nappi O, Glasner SD, Swanson PE, Wick MR. Biphasic and monophasic sarcomatoid carcinomas of the lung: a reappraisal of 'carcinosarcomas' and 'spindle-cell carcinomas'. Am J Clin Pathol 1994; 102:331-40.

3. Jang KS, Jang SH, Oh YH, Choi HS, Lee KG, Choi D, et al. Sarcomatoid carcinoma of the distal common bile duct: a case report. J Pathol Transl Med 2005:39:360-3.

4. Lopez-Beltran A, Pacelli A, Rothenberg HJ, Wollan PC, Zincke H, Blute ML, et al. Carcinosarcoma and sarcomatoid carcinoma of the bladder: clinicopathological study of 41 cases. J Urol 1998;159:1497503 .

5. Okabayashi T, Sun ZL, Montgomey RA, Hanazaki K. Surgical outcome of carcinosarcoma of the gall bladder: a review. World J Gastroenterol 2009; 15:4877-82.

6. Loud PA, Warshauer DM, Woosley JT, Hartmann TM. Carcinosarcoma of the extrahepatic bile ducts: cholangiographic and CT appearance. Abdom Imaging 1997;22:85-6.

7. Yoon GS, Choi DL. Sarcomatoid carcinoma of common bile duct: a case report. Hepatogastroenterology 2004;51:106-9.

8. Kadono J, Hamada N, Higashi M, Ishizaki N, Nakamura N, Sakata R. Carcinosarcoma of the extrahepatic bile duct. J Hepatobiliary Pancreat Surg 2005;12:328-31. 
9. Sodergren MH, Silva MA, Read-Jones SL, Hubscher SG, Mirza DF. Carcinosarcoma of the biliary tract: two case reports and a review of the literature. Eur J Gastroenterol Hepatol 2005;17:683-5.

10. Aurello P, Milione M, Dente M, D’Angelo F, Nigri G, Del Gaudio $\mathrm{M}$, et al. Synchronous carcinosarcoma of the intrapancreatic bile duct and carcinoma in situ of wirsung duct: a case report. Pancreas 2008;36:95-7.

11. Tanaka M, Ajiki T, Matsumoto I, Asari S, Fukumoto T, Masuda A, et al. Duodenal protrusion by carcinosarcoma of the extrahepatic bile duct. Dig Endosc 2012;24:484.

12. Kumei S, Onishi Y, Ogura T, Kusumoto C, Matsuno Y, Nishigami T, et al. Carcinosarcoma of the extrahepatic bile duct presenting with stone-like radiological findings. Intern Med 2015;54:1747-51.

13. Lee SY, Shia J, Kingham TP, Jarnagin WR. Carcinosarcoma of the bile duct: a case report and review of literature. Hepatobiliary Surg Nutr 2016;5:72-8.

14. Zhang S, Jia J, Bi X, Jiang Q, Zhao Y, Chen Y, et al. Sarcomatoid carcinoma of the common bile duct: a case report. Medicine (Baltimore) 2017;96:e5751.

15. Wong MW, Chen MJ, Chen CJ, Hung CY, Wang HY, Chang CW. Gallbladder sarcomatoid carcinoma: a case report and review of current literature. Adv Dig Med 2016;3:61-6. 